\title{
Skin marker placement by technologist prior to knee MRI helps identify clinically relevant pathologies
}

\author{
Vibhor Wadhwa' ${ }^{1}$ Eric Weissman², Daichi Hayashi, ${ }^{3,4}$, Yin Xi $^{2}$ and Avneesh Chhabra ${ }^{2,5,6^{*}}$
}

\begin{abstract}
Background: Majority of musculoskeletal cross-sectional imaging requests have a non-revealing and non-specific clinical history of pain. However, the location of pain is very relevant towards arriving at a specific orthopedic diagnosis. The purpose of this research was to study the impact of skin marker placement and training of technologists prior to knee MRI in detection of clinically important findings.

Methods: Total 200 consecutive left knee MRIs were evaluated before and after technologist training with regards to marker placement at the site of clinical symptoms or palpable finding. Marker location in relation to the knee was recorded and important findings were classified as correlated important finding, non-correlated important finding, other compartment important finding in non-correlated cases, and diffuse abnormality, i.e. tri-compartmental cartilage defects in both correlated and non-correlated cases. Differences among scans before and after technologist training were analyzed.
\end{abstract}

Results: The marker placement was observed in higher proportion of patients in post-training scans (78\% vs $60 \%, p=0.00$ ). The most common location of the marker was in anterior or anterolateral knee (32\% and $34 \%$ cases, respectively). The marker-important finding correlation was also higher post training, but not statistically significant (53\% versus 38\%, $p=0.57$ ). Important findings correlated with the marker in more than $50 \%$ of the scans in the post-training set.

Conclusion: Marker placement can aid in detection of clinically important imaging finding and technologist training aids in increased rates of marker placement and improved correlation.

Keywords: Knee MRI, MRI skin marker, Meniscus tear, Ligament tear, Cartilage defect

\section{Background}

In our tertiary care practice, majority of orthopedic cross-sectional study orders have a non-revealing and non-specific (in terms of location) history of pain. However, it is well known that site of pain e.g. joint line tenderness is quiet relevant in arriving at diagnosis of orthopaedic conditions, such as meniscus tear $[1,2]$. Similarly, many joint diagnoses based on history and examinations reflect the site of patient's symptoms. Musculoskeletal radiologists reading these joint MRIs and particularly those using a structured reporting

\footnotetext{
* Correspondence: avneesh.chhabra@utsouthwestern.edu

2Department of Radiology, UT Southwestern Medical Center, Dallas, TX, USA

${ }^{5}$ Department of Orthopaedic Surgery, UT Southwestern Medical Center, 5323

Harry Hines Blvd, Dallas, TX 75390-9178, USA

Full list of author information is available at the end of the article
}

template find many related and unrelated findings with respect to the patient's presentation. The radiologist may not have access to the patient's chart during study readout or even with chart access, extracting relevant history and examination takes time and reduces the efficiency of the radiologist in busy practice environments.

In majority of radiology practices, the radiologist often does not see the patient, and the only point of contact for the patient is the technologist. The practice of communicating patient's clinical information from the technologist to the radiologist vary from onsite documentation of patient history in the chart, attaching the referring physician's note to the patient images, to direct site marking by the technologist before the examination. The musculoskeletal department at our institution 
adopted the policy of onsite marker placement at the site of concern, i.e. "most painful or only painful site" and/or palpable swelling. The purpose of this study was:

1. To evaluate the correlation of skin marker placement by the technologist to an important clinical finding, and

2. Study the impact of re-training of technologists on frequency of marker placement and correlation of marker site post-training with the underlying pathology.

\section{Methods}

Institutional board review approved retrospective study was performed and informed consent was waived for this HIPAA compliant quality improvement (QI) project. 200 consecutive knee MRIs in patients were evaluated in two groups - before and after technologist training with regards to skin marker (MR-SPOT, $5.0 \mathrm{~cm}$, Beekley Medical Corporation, Fig. 1) placement before the Knee MRI. Both pre-and post-training groups encompassed 100 scans each. Only left knee MRIs were included in the study to maintain consistency. The MRIs were evaluated for the frequency of marker placement by a musculoskeletal radiology fellow. The marker location in relation to the knee was categorized as: anterior, posterior, anterolateral, anteromedial, medial and lateral. The important imaging findings evaluated included: medial or lateral meniscus tear, medial or lateral collateral ligament tear, anterior or posterior cruciate ligament tear,

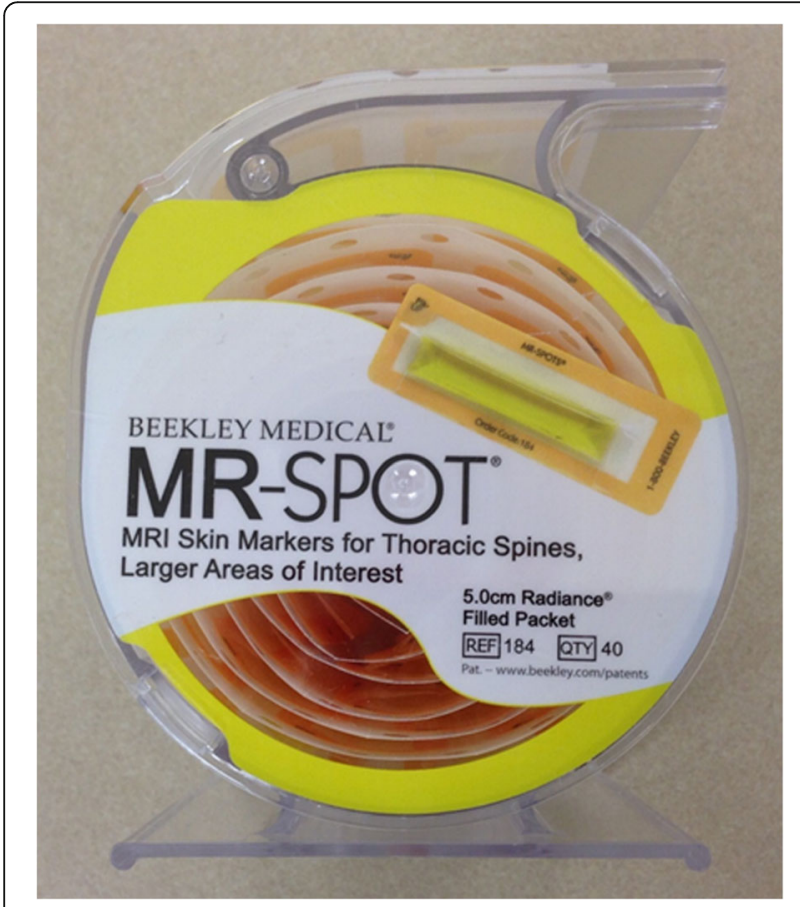

Fig. 1 MR-SPOT MRI skin marker (Beekley Medical Corporation) high grade or full-thickness cartilage defects and moderate-large effusion. Patient demographics, presenting complaint and post-imaging treatment were also recorded. The imaging findings were classified as follows:

- corroborative and important, i.e. finding correlated with location of the skin marker. e.g. a medial meniscus tear in the setting of a medial marker.

- non-corroborative important, i.e. finding did not correlate with location of the skin marker, e.g. a lateral meniscus tear in the setting of a medial marker.

- other compartment important finding in corroborative cases, i.e. lateral and medial meniscus tears in a medial marker.

- diffuse abnormality, i.e. tricompartmental cartilage defects in both corroborative and -non-corroborative cases.

Differences among scans before and after the technologist training were assessed using chi-square test for categorical variables and $\mathrm{t}$-test for continuous variables.

\section{Results}

The results are summarized in Table 1. The patient demographics in both groups of scans were not statistically different (age $p=0.2$ and sex $p=0.3$ ). Chief complaint in majority of patients in both groups was non-specific pain

Table 1 Patient demographics and impact of marker placement in pre- and post-training groups

\begin{tabular}{|c|c|c|c|}
\hline & $\begin{array}{l}\text { Pre-Training } \\
\text { Group }\end{array}$ & $\begin{array}{l}\text { Post-Training } \\
\text { Group }\end{array}$ & $P$ value \\
\hline Number of MRIs & 100 & 100 & \\
\hline Age & & & 0.2434 \\
\hline Mean & 48.05 & 50.37 & \\
\hline SD & 14.87 & 13.12 & \\
\hline Sex & & & 0.3133 \\
\hline Female & 56 & 63 & \\
\hline Male & 44 & 37 & \\
\hline Clinician History & & & 0.1649 \\
\hline Pain & 75 & 83 & \\
\hline Other & 25 & 17 & \\
\hline Marker Present & & & 0.0059 \\
\hline Yes & 60 & 78 & \\
\hline No & 40 & 22 & \\
\hline Marker-Finding Correlation & & & 0.5706 \\
\hline Yes & 38 & 53 & \\
\hline No & 22 & 25 & \\
\hline Diffuse Findings & & & 0.004 \\
\hline Yes & 14 & 31 & \\
\hline No & 86 & 69 & \\
\hline
\end{tabular}




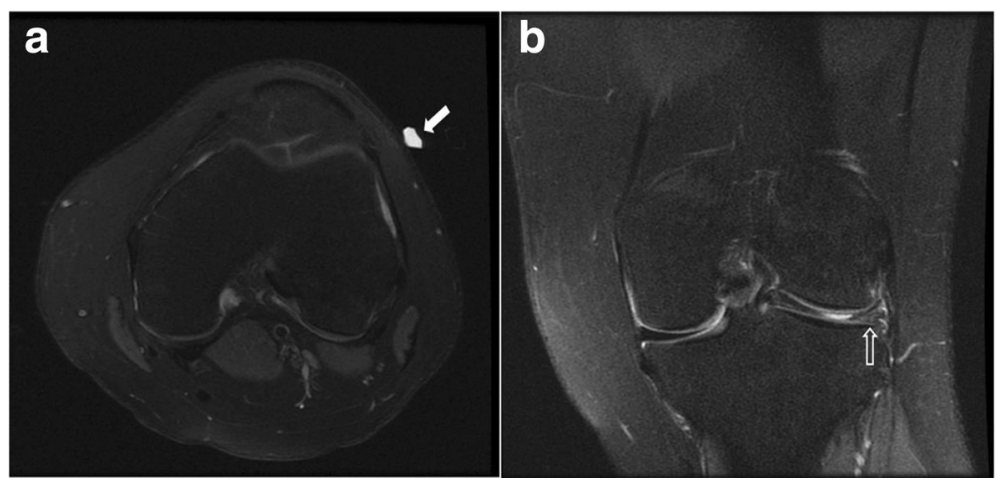

Fig. 2 Lateral marker (solid arrow in a) and corroborative finding of bucket handle tear in the lateral meniscus (open arrow in b)

(75\% in pre-training group, $83 \%$ in post-training group, $p=0.16)$. Marker was observed in higher number of scans in the post training group (78\% vs $60 \%$, $p=0.00)$. Most common location of the marker was in anterior or anterolateral sites (32\% and 34\% cases, respectively). Corroborative and important findings were found to be higher, but not statistically different, in the post-training group (53\% vs $38 \% ; p=0.57)$ (Figs. 2, 3 and 4). However, diffuse abnormalities were more prevalent in post-training group $(31 \%$ vs $14 \%, p=0.04)$, which may partially explain the statistical non-significance. Other associated findings in pre- and post-training groups (14\% vs $13 \%)$ included medial meniscus tears (3 vs 7), lateral meniscus tears (3 vs 2), ACL tears (5 vs 1 ), MCL tear ( 1 vs 0 ), PCL tear ( 0 vs 1 ), and other compartment arthritis (2 vs 1$)$. Majority of patients received conservative management (90\%).

\section{Discussion}

MRI-compatible skin markers have been used as a preoperative guidance tool for neurosurgeons in brain and spinal surgeries [3]. Fischer et al. described use of a special skin marker and inserted a nonmagnetic wire into the breast to achieve MRI-guided localization of suspected breast lesions. Repeat diagnostic MRI was performed to document the position of the wire tip relative to the lesion, to allow successful excision [4]. Besides commercially available markers, fish oil capsules have been used as MRI localization devices as a cost-effective alternative [5]. However, pre-scan application of skin marker in musculoskeletal settings has not been scientifically studied yet.

The present study shows that skin marker location highly correlates with important clinical findings, i.e. in upto $53 \%$ cases and thus, placement before knee MRI can potentially help the radiologist in producing clinically relevant reports. In routine reading of high volume of knee MRI studies and using structured template reporting, one may detect a number of imaging findings which could be unrelated to the patient's symptoms, and may divert the attention of the radiologist from the area of interest. The clinical indication presented to the radiologist for joint MRI is usually non-specific (non-

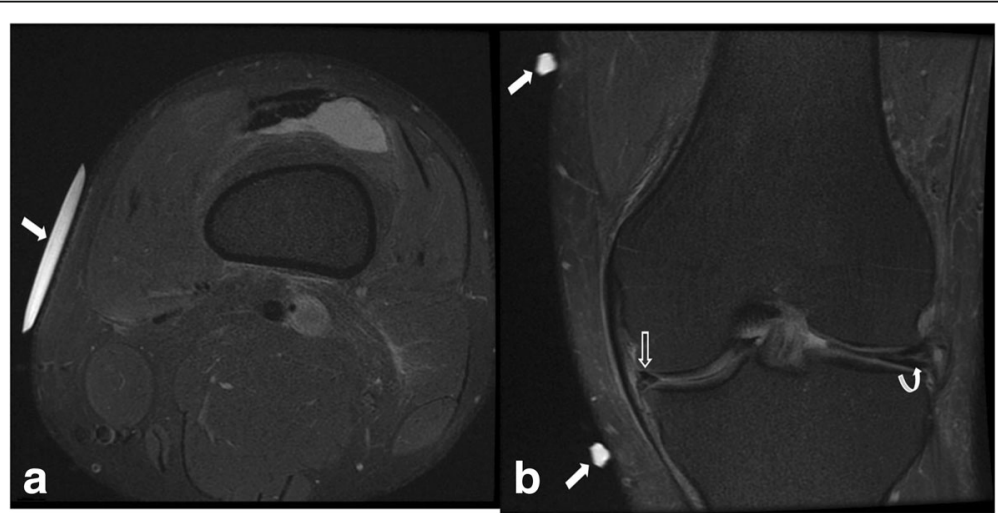

Fig. 3 Medial marker (solid arrows) and corroborative finding of medial meniscal bucket handle tear (open arrow in b). Also note there is a lateral meniscal horizontal tear (curved arrow in b). Axial (a) and coronal (b) images 


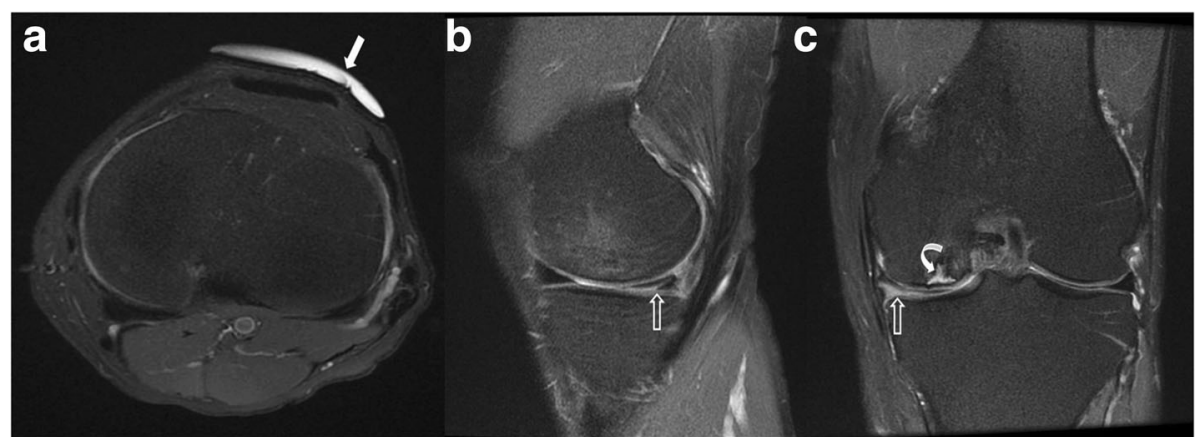

Fig. 4 Anterior marker (solid arrow) and non-corroborative finding of medial meniscal tear (open arrows) and subchondral fracture (curved arrow). Axial (a), sagittal (b) and coronal (c) images

localizing) pain, as observed in the majority of cases in present study as well. Since there is limited clinical information available to the radiologist, skin markers can help in identification of pathology with increased confidence. Finding clinically correlated important internal knee derangement lesions in more than $50 \%$ cases by just using an external marker is a diagnostically relevant result. It should also be noted that in $47 \%$ cases, there were other findings, such as cruciate ligament injuries and non-correlated other compartment derangements. Therefore, comprehensive evaluation is still warranted during knee MRI reporting. However, the marker placement may help the radiologist rank the impressions more relevant to the clinical scenario and presentation.

Our study also shows that training of the technologists in application of knee markers at the area of interest, i.e. point of maximum pain or swelling, increases the frequency of marker placement. This cements the fact that, indirectly, active intervention of radiologist can keep up the interest of the technologists in quality improvement of imaging. Furthermore, the correlation of markerimportant finding is also improved with technologist training and they become better at marking the clinically important site of abnormality.

We also found important imaging finding in 13-14\% cases unrelated to the marked site. Therefore, although marking seems important and correlated finding should be put as the first impression, interrogation of all compartments of the joint remains essential.

There are some limitations to our study. First, this was a retrospective study and not a prospectively derived cohort. Second, we did not obtain interobserver performance since formal reports were available for all the studies and this is the 1st exploratory study in this domain.

In future, similar studies in other joints, such as wrist, ankle, shoulder and hip could be performed to further validate the usefulness of marker placement. Time of imaging evaluation can be assessed with and without marker in detection of clinically important finding. A similar concept can be expanded to other imaging modalities, such as abdominal CTs, which are performed on a large scale, especially on call. It might help the reader to detect the important imaging finding quickly and consistently.

\section{Conclusion}

Skin marker placement can aid in detection of clinically important imaging finding and technologist training aids in increased rates of marker placement and improved correlation. Therefore, marker placement can potentially improve the clinical relevance of knee MRI reports.

Abbreviations

MRI: Magnetic Resonance Imaging; Ql: Quality Improvement

\section{Acknowledgements}

None

Funding

No funding was obtained for this study.

Availability of data and materials

Data is available upon request via email.

\section{Disclosures}

Avneesh Chhabra serves as a consultant with Siemens for MSK CAD group and ICON medical. He also receives royalties from Jaypee and Wolters Kluwer. All authors report no conflict of interest.

\section{Authors' contributions}

WW: study concepts and design, literature search, manuscript preparation. EW: study concepts and design, manuscript preparation. DH: study concepts and design, manuscript editing. YX: statistical analysis, manuscript editing. AC: guarantor of integrity of the entire study, study concepts and design, manuscript editing. All authors read and approved the final manuscript.

Ethics approval and consent to participate

Institutional review board of UT Southwestern approved this for this HIPAA compliant quality improvement (QI) project and informed consent was waived due to its retrospective nature.

Consent for publication Not Applicable 


\section{Competing interests}

Dr. Avneesh Chhabra and Dr. Daichi Hayashi serve as members of editorial board of BMC Musculoskeletal Disorders.

\section{Publisher's Note}

Springer Nature remains neutral with regard to jurisdictional claims in published maps and institutional affiliations.

\section{Author details}

'Department of Radiology, University of Arkansas for Medical Sciences, Little Rock, AR, USA. ${ }^{2}$ Department of Radiology, UT Southwestern Medical Center, Dallas, TX, USA. ${ }^{3}$ Department of Radiology, Boston University School of Medicine, Boston, MA, USA. ${ }^{4}$ Department of Radiology, Stony Brook Medicine, Stony Brook, NY, USA. ${ }^{5}$ Department of Orthopaedic Surgery, UT Southwestern Medical Center, 5323 Harry Hines Blvd, Dallas, TX 75390-9178, USA. ${ }^{6}$ Adjunct faculty, Department of Radiology, Johns Hopkins University, Baltimore, USA.

Received: 29 July 2017 Accepted: 24 November 2017

Published online: 15 December 2017

\section{References}

1. Eren OT. The accuracy of joint line tenderness by physical examination in the diagnosis of meniscal tears. Arthroscopy. 2003;19(8):850-4.

2. Hing W, White S, Reid D, Marshall R. Validity of the McMurray's test and modified versions of the test: a systematic literature review. J Man Manip Ther. 2009:17(1):22-35.

3. Rosahl SK, Gharabaghi A, Liebig T, Feste CD, Tatagiba M, Samii M. Skin markers for surgical planning for intradural lesions of the thoracic spine. Technical note. Surg Neurol. 2002;58(5):346-8.

4. Fischer U, Vosshenrich R, Bruhn H, Keating D, Raab BW, Oestmann JW. MRguided localization of suspected breast lesions detected exclusively by postcontrast MRI. J Comput Assist Tomogr. 1995;19(1):63-6.

5. Gilbert JW, Wheeler GR, Richardson GB, Herder SL, Mick GE, Gyarteng-Dakwa K, et al. Guidance of magnetic resonance imaging and placement of skin-marker localization devices. J Neurosurg Sci. 2011;55(2):85-8.

Submit your next manuscript to BioMed Central and we will help you at every step:

- We accept pre-submission inquiries

- Our selector tool helps you to find the most relevant journal

- We provide round the clock customer support

- Convenient online submission

- Thorough peer review

- Inclusion in PubMed and all major indexing services

- Maximum visibility for your research

Submit your manuscript at www.biomedcentral.com/submit
Biomed Central 